\title{
Calcinosis tumoral secundaria a enfermedad renal crónica
}

\author{
Tumoral calcinosis secondary to chronic kidney \\ disease
}

\author{
Gustavo Andrés Ortega, Diego luis SaAibi, Andrés Mauricio Olarte, \\ Miguel Reinaldo Mora, Gustavo Eduardo Ortega • Bucaramanga (Co- \\ LOMBIA)
}

\section{Resumen}

Objetivo: evaluar la literatura disponible hasta hoy, respecto a la calcinosis tumoral en enfermedad renal crónica, centrándose en herramientas diagnósticas y manejo terapéutico disponible.

Metodos: usando los siguientes descriptores "Calcinosis", "Kidney Failure, Chronic”, "Therapeutics", "Diagnosis", "Epidemiology"; se buscaron artículos en la base de datos Pubmed. Se incluyeron artículos de ensayo clínico, metaanálisis, revisiones sistemáticas y casos clínicos que estuvieran en inglés, francés, portugués y español, que fueran en población adulta. Se excluyeron artículos publicados antes de 1990, excepto aquellos que fuesen vitales para la definición de calcinosis tumoral. Se excluyeron artículos que incluyeran "Calciphylaxis" y que no aportaran al objetivo de la revisión.

Resultados: un total de 91 artículos fueron incluidos, la mayoría fueron casos clínicos. Según lo encontrado el sitio de calcinosis tumoral más común fue en miembros superiores, seguido de piel y cadera. Una de las recomendaciones más importantes de diagnóstico fue las imágenes radiográficas y la química sanguínea, y de tratamiento es el uso de quelantes de fósforo combinado con dieta baja en calcio y fósforo.

Conclusión: se incluyó gran cantidad de información. Faltan más estudios en Latinoamérica respecto a esta condición y se necesitan mejores grados y calidad de evidencia para hacer algún tipo de recomendación o guía de manejo. (Acta Med Colomb 2012; 37: 74-79)

Palabras clave: calcinos, enfermedad renal, crónica, diagnóstico, tratamiento, revisión sistemática.

\section{Abstract}

Objective: to assess the available literature to date regarding tumoral calcinosis in chronic renal disease, focusing on diagnostic tools and therapeutic management available.

Methods: using the descriptors "Calcinosis", "Kidney Failure, Chronic", "Therapeutics", "Diagnosis", "Epidemiology", we sought articles in the pubmed database. We included clinical trial articles, meta-analyzes, systematic reviews and clinical cases that were in English, French, Portuguese and Spanish, all in adults. We excluded articles published before 1990 except those that were vital to the definition of tumoral calcinosis. Articles that included "Calciphylaxis" and that did not contribute to the objective of the review, were excluded.

Results: a total of 91 articles were included; most were case reports. We found that the most common site of tumor calcinosis was in the upper limbs, followed by skin and hip. One of the key diagnostic recommendations was radiographic images and blood chemistry, and the recommendation regarding treatment is the use of phosphorus binders combined with diet low in calcium and phosphorus.

Conclusion: we included a lot of information. Further studies on this condition are needed in Latin America, as well as better grades and quality of evidence to make any recommendation or management guide. (Acta Med Colomb 2012; 37: 74-79)

Keywords: calcinosis, kidney disease, chronic, diagnosis, treatment, systematic review.
Dr. Gustavo Andrés Ortega Ramírez: Médico Especialista en Medicina Interna, Universidad Metropolitana, Barranquilla; Andrés Mauricio Olarte M: Estudiante, Programa de Medicina, Universidad Autónoma de Bucaramanga (UNAB), Bucaramanga; Dr. Miguel Reinaldo Mora: Médico Especialista en Medicina Interna, Universidad Metropolitana, Barranquilla; Gustavo Eduardo Ortega Ramírez: Estudiante, Programa de Medicina, Universidad de Santander (UDES), Bucaramanga (Colombia). Correspondencia. Dr. Gustavo Andrés Ortega.

E-mail: gustavito207@hotmail.com Recibido: 08/VIII/2011 Aceptado 16/V/2012 


\section{Introducción}

La calcinosis tumoral (CT) fue descrita en un inicio por Girad y Duret en $1898(1,2)$, pero no fue sino hasta 1949 que Teutschlaender y cols. le dieran una definición al término y le apodaran la 'enfermedad de lipogranulomatosis o de Teutschlaender'(3). Más recientemente Smack y cols. propusieron una clasificación para la CT basándose en la etiopatogenia, esta fue: 'calcinosis tumoral familiar normofosfatémica', 'calcinosis tumoral familiar hiperfosfatémica' y calcinosis tumoral secundaria (p. ej. a enfermedad renal crónica) (4). A continuación presentamos una revisión sistemática de la literatura relacionada con la calcinosis tumoral secundaria a enfermedad renal crónica con el objetivo de conocer qué intervenciones existen para el diagnóstico y el tratamiento de la calcinosis tumoral secundaria a enfermedad renal crónica.

\section{Método de búsqueda}

En la base de datos Pubmed, se realizó una búsqueda incluyendo literatura hasta la fecha 30/7/2011. Se utilizaron los siguientes descriptores para la búsqueda: "Calcinosis"[Mesh], "Kidney Failure, Chronic"[Mesh], "Therapeutics"[Mesh], "Diagnosis"[Mesh], "Epidemiology" [Mesh]. La búsqueda arrojó 1083 artículos y su filtración con criterios de exclusión se describen en la Figura 1. Al final del filtro de artículos quedaron 91 bibliografías, la mayoría casos clínicos.

\section{Definición}

La calcinosis tumoral (CT) es un término que comprende CT familiar, y CT secundaria (4). Otra definición más reciente dice que representa un grupo heterogéneo de trastornos genéticos, hereditarios, o inherente a un desorden que se

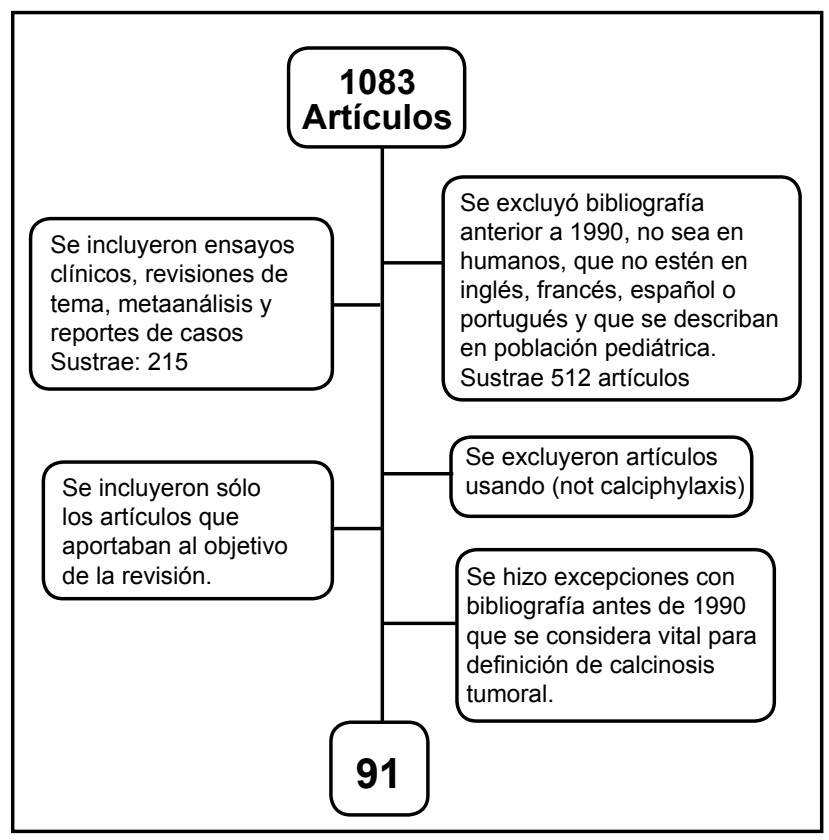

Figura 1. Diagrama de flujo para la filtración de artículos. manifiesta con depósitos de material calcificado en tejido cutáneo y subcutáneo que se localiza generalmente en las áreas periarticulares de grandes articulaciones (5).

\section{Fisiopatología}

La fisiopatología de la calcinosis tumoral no ha sido dilucidada pero se han propuesto diferentes mecanismos. Éstos son:

A) Traumas repetitivos que inducen a una disfunción en la reparación del tejido.

B) Disección de fuerzas periarticulares, con agregados histiocíticos, que conllevan a una activación osteoclástica.

C) Hemorragias ocasionadas por microtraumas, causando una respuesta exagerada de reparación (6).

Como CT secundaria a enfermedad renal crónica se ha propuesto que un valor de fosfato de calcio sérico elevado, producto de un $\mathrm{pH}$ y un magnesio sérico elevado así como injurias locales pueden desencadenar una pronunciada calcificación de los tejidos blandos. Estrictamente en el contexto de la enfermedad renal crónica esto es explicado por una alteración del metabolismo del fosfato de calcio asociado a un hiperparatiroidismo secundario con hiperfosfatemia (7-10).

Se ha asociado también a esta entidad el uso de vitamina D activa y calcio con quelantes de fósforo a esta condición (11). Genéticamente hablando, se ha podido hallar cierta relación entre algunos genes y la CT. En la calcinosis familiar normofosfatémica se han encontrado defectos en mecanismos de la apoptosis, por mutaciones en: a) galnt3, b) gen que codifica el fgf 23, c) kl, d) samd9 (5).

El galnt 3 es un gen que codifica una glicosil transferasa, denominada también uridina difosfato- n- acetil galactosamina; cataliza el primer paso de la o - glicosilación de la serina y residuos de treonina (12).

El fgf 23 es una proteína fosfatúrica, responsable de promover la excreción de fósfato por el riñón.

Klotho $(\mathrm{kl})$ es el receptor del factor de crecimiento fibroblástico implicado en la patogenia.

Samd9 es una proteína de degradación y se ha postulado que esta proteína está involucrada en la inducción del factor de necrosis tumoral alfa, desencadenando la apoptosis celular.

El funcionamiento defectuoso de cualquiera de éstos, puede resultar en hiperfosfatemia y aparición de calcificaciones ectópicas $(13,14)$. Lo anotado indica la importancia de cada uno de estos genes, en lo que sería, la prevención de calcificaciones anormales de tejidos en zonas periféricas, planteándose la posibilidad de evaluar morbilidad y mortalidad, con presencia de calcificaciones extraóseas (vasculares o cardiovasculares). La fracción de excreción del fosfato es entre el 5 y el $20 \%$, depende del balance funcional del calcio y el hidrógeno. El fosfato en su mayor parte es reabsorbido en el túbulo proximal, por un simportador sodio-fosfato tipo 3. La regulación y expresión de este transportador también depende del nivel de paratohormona; este mecanismo es el que se altera, se adiciona alcalosis metabólica y una injuria 
tisular que desencadene el proceso y esto hasta ahora se mantiene como teoría predominante en la fisiopatología de la CT secundaria a enfermedad renal crónica.

\section{Manifestaciones y diagnóstico}

En esta revisión sistemática se encontró gran cantidad de información proveniente de series de casos y reportes de casos observándose que la manifestación más frecuente de calcinosis tumoral es en miembros superiores seguida de la piel. Las localizaciones de depósito de las sales de calcio se exponen en la Tabla 1.

Como se puede observar en la Tabla 1, la mayoría de casos corresponden a CT en miembros superiores entre hombro, codo y muñeca. Además podemos observar que otras sitios anatómicos menos frecuentes como el cuello, corazón y SNC pueden ocasionar consecuencias muy graves dentro del contexto de una condición que se cree benigna o de pocas implicaciones más allá de las masas protuyentes. Por ejemplo se han visto casos donde el compromiso de los depósitos ejercidos sobre la médula espinal han generado hemiparesias y tetraparesias reversibles (78). Además de las lesiones que se observan en mama secundarias a la calcinosis tumoral, se ha visto cómo ésta y otras calcificaciones han disminuido la precisión de la mamografía en diagnosticar el cáncer y además pueden confundir a los evaluadores y dar un críterio errado que podría retardar un diagnóstico con sus complicaciones subyacentes (35). Otro tipo de manifestación puede ser la compresión de diferentes nervios como el ciático cuando la masa se ubica en cadera y el nervio ulnar cuando se comprime la fosa de Guyon (54). En un hecho extremadamente inusual se reportó un caso en

Tabla1. Recopilación de los sitios anatómicos más frecuentes de la calcinosis tumoral.

\begin{tabular}{|l|c|c|}
\hline Sitio anatómico & Número de casos hallados & Referencias* \\
\hline Abdomen & 7 & $15-21$. \\
Cabeza (no SNC) & 2 & 22,23 \\
Cadera & 9 & $7,8,24-30$. \\
Cervical & 3 & $10,30,78$ \\
Difusa & 1 & 32 \\
Hepática & 1 & 33 \\
Mamas & 4 & $31-36$ \\
Miocardio & 7 & $37-43$ \\
MiInf & 4 & $44-48$ \\
MiSs & 14 & $7,11,15,26,49-58$. \\
Ojo & 10 & 59 \\
Piel (cutis) & 7 & $75-77$ \\
Pulmón & 3 & $50,57,70-74$ \\
SNC (encéfalo y espinal) & \multicolumn{2}{|c|}{} \\
\hline $\begin{array}{l}\text { Algunas referencias pueden contribuir con más de un sitio anatómco ya que se tratan de } \\
\text { más de un caso o un mismo caso con CT en varias partes del cuerpo. MiInf: Miembros }\end{array}$ \\
inferiores. MiSs: Miembros superiores.(esta tabla fue creada por los mismos autores, \\
a partir de cada referencia según el orden que le adjudicamos) \\
\hline
\end{tabular}

que la manifestación fue una embolización hacia miembros inferiores de material calcificado proveniente de una CT en el miocardio de ventrículo izquierdo (41), esto supone pensar que aunque infrecuente una CT en corazón es un nuevo riesgo de embolismo en pacientes crónicos renales en diálisis. Poco se conoce respecto a nuevas técnicas imagenológicas para diagnosticar la CT. Desde hace varios años la técnica más usada sigue siendo la radiografía convencional. En la radiografía simple se puede ver la masa calcificada de un tamaño considerable, con su caracteristica forma en 'guijarro' o redondeada, radiopaca con paredes fibrosas radiolúcidas (signo de "alambre de pollo") (79). Recientemente se ha observado que las imágenes por captación de radionúclidos o escintigrafías usando tecnecio-99m-etileno difosfonato (Tc-99m MDP) revelan de manera temprana los tejidos con pequeños depósitos calcificados (generalmente dihidroxiapatita) y se ha usado ampliamente en pacientes crónicos renales con resultados esperanzadores que permiten un mejor seguimiento de las lesiones pero que no previene su aparición (80-82). Las imágenes por tomografía computarizada han permitido observar con más detalle este tipo de lesiones; se ha descrito un signo de sedimentación donde se aprecia la masa como una estructura semisólida; la reconstrucción en imágenes $3 \mathrm{D}$ ha permitido una extraordinaria visualización de las lesiones (Figura 2).

\section{Tratamiento}

Aunque se han reportado que algunas lesiones de CT en pacientes con falla renal hacen regresión espontánea $(83,84)$, no es el caso de la mayoría de las lesiones. A continuación

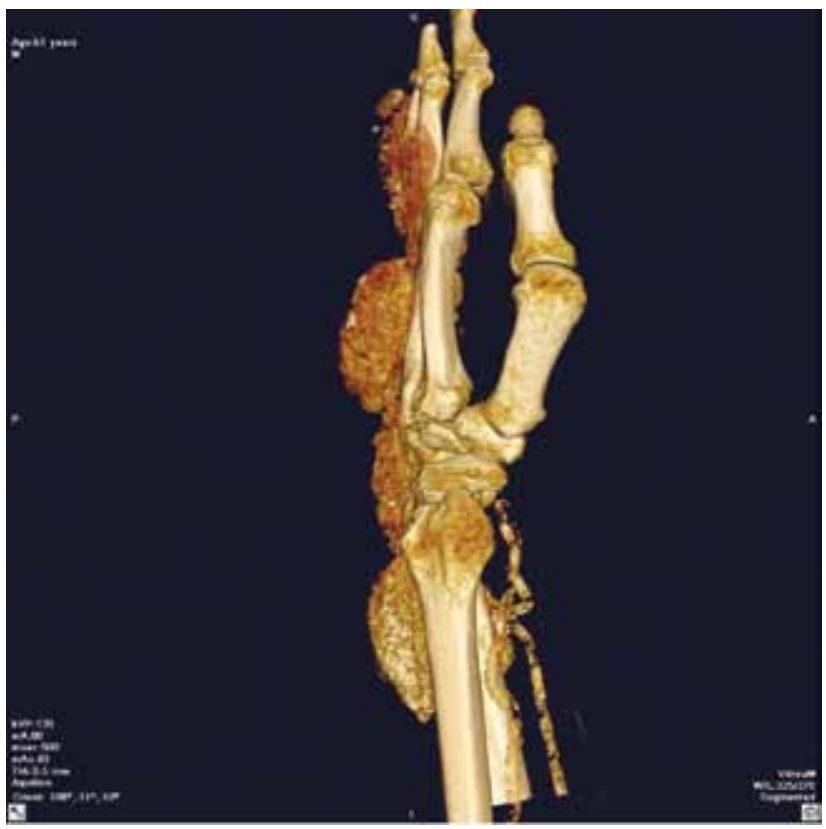

Figura 2. Una vista lateral de mano izquierda que muestra el compromiso de las lesiones y su clásica apariencia de "canto rodado" o "guijarro". Si se observa detenidamente se pueden ver los depósitos de calcio en los vasos radial y ulnar. (Adjunto el consentimiento informado del paciente). 
se describen las alternativas terapéuticas farmacológicas y no farmacologicas encontradas. Cinacalcet, un agonista de los canales de calcio, se usa en el tratamiento del hiperparatiroidismo secundario y se ha probado en un ensayo clínico como alternativa para la terapia de calcinosis tumoral (85). Una alternativa no farmacológica que sirve para disminuir la tasa de CT en pacientes con hemodiálisis probada por un ensayo clínico aleatorizado es realizar las hemodiálisis de manera nocturna y no matutina, como se hace convencionalmente. Se ha visto que esta práctica disminuye los niveles de fósforo sérico, fosfato de calcio y la necesidad de uso de quelantes de fósforo, lo que por ende disminuye las tasas de CT y otros trastornos metabólicos en los paciente crónicos renales con diálisis (86).

El uso de bifosfonatos se ha descrito en la CT. El mecanismo de acción de los bifosfonatos es la inhibición de la resorción ósea por los osteoclastos. Además disminuye las citoquinas proinflamatorias como IL-1 y factor de necrosis tumoral alfa. Dosis diarias de pamidronato $(30 \mathrm{mg}) \mathrm{se}$ administraron tres días consecutivos con el fin de parar la actividad osteoclástica (87). El etidronato ha sido reportado en el uso de la CT usándolo por dos años en un paciente que no había respondido al tratamiento quirúrgico. Se usó el etidronato disódico $400 \mathrm{mg}$ por 14 días en ocho ciclos distribuido en dos años, lo que redujo radiológicamente y clínicamente las lesiones de CT. El tratamiento no tuvo efectos adversos (88). El alendronato ha sido utilizado para esta patología en dosis de $70 \mathrm{mg}$ semanales, por periodos hasta de 15 meses, que disminuye las lesiones, y se asocia con altas tasas de recidiva (89). Otros autores describen como el uso de aluminio con quelantes de fósforo, dieta baja en calcio y fósforo y hemodiálisis logrando disminuir el tamaño de la lesión en la muñeca de un paciente con enfermedad renal terminal en un periodo de dos meses. El tratamiento radical aunque con gran tasa de recidivas es la terapia quirúrgica combinada con la deprivación de fosfato, con hidróxido de alumnio, junto con acetazolamida, de forma sinérgica ha demostrado ser una terapia eficaz. El tratamiento quirúrgico se aplica para evitar también, la aparición de complicaciones (fístulas y compresiones nerviosas) y por razones cosméticas. La exéresis debe ser meticulosa y completa, pues la recidiva es especialmente alta, luego de una escisión incompleta. En el caso de la tendinitis se ha demostrado beneficios, por medio de múltiples estudios aleatorizados, con terapia de ultrasonido (90). La terapia dirigida para las calcificaciones de los desórdenes metabólicos, se basa en tratar la causa subyacente del trastorno metabólico, por ejemplo terapia dialítica para los pacientes con enfermedad renal crónica, osteodistrofia y la regulación hormonal en el contexto de un hiperparatiroidismo primario (90). Cuando se asocia la CT a un hiperparatiroidismo se puede considerar la paratiroidectomía parcial o total en tanto se halla fallado con el tratamiento de dieta y quelantes de fósforo. Las tetraciclinas han demostrado mejorar el curso de las calcinosis, relacionándose con la capacidad de inhibir metaloproteinasas, que puede desempeñar un papel importante en la patogénesis (91).

\section{Conclusiones}

La presente revisión sistemática reunió gran cantidad de información disponible en la red más grande de bibliografía médica disponible. Desafortunadamente pocos reportes o estudios latinoamericanos se incluyeron en la presente revisión debido a la dificultad que supone la búsqueda tal cual como se planteó, en las bases Redalyc, Scielo, Lilacs, no hay indexados artículos que se crucen con la metodología de búsqueda específica usada en la presente revisión sistemática; no obstante la información obtenida, es muy valiosa para tener conceptos claros y que el cuerpo de salud que atiende la población con enfermedad renal crónica sospeche en un paciente con características de CT y tenga alguna guía de cómo se debe proceder. Para el diagnóstico y la terapia de la CT se debe concluir que: un paciente con enfermedad renal crónica con tumoraciones de consistencia pétrea pronunciadas con más frecuencia en miembros superiores, piel o cadera, se debe proceder con una radiografía convencional, revisar si las lesiones tienen las características comunes de la CT. Si el paciente refiere dolor $\mathrm{u}$ otras condiciones que puedan ser consecuencia de la CT se puede evaluar con una TAC. Si se realiza el diagnóstico de $\mathrm{CT}$, se le debe realizar en el paciente un completo chequeo de química sanguínea incluyendo calcio, fósforo, paratohormona, sin olvidar la tasa de filtración glomerular. Una vez obtenidos estos parámetros se puede proceder con quelantes de fósforo, optimizar los tiempo de diálisis y si es posible realizarlos en horas nocturnas combinado con una dieta baja en calcio y en fósforo (esto no es posible en niños en creciemiento).

Se puede considerar el uso de bifosfonatos como el alendronato, pamidronato y etidronato, pero hay que tener precaución debido a que su uso y su efectividad aún no han sido estudiadas por al menos un ensayo clínico para la CT. $\mathrm{Si}$ en un periodo de dos meses no se ve mejoría clínica o radiológica de las lesiones se puede considerar una paratiroidectomía subtotal. La resección de las lesiones de la $\mathrm{CT}$ se debe tomar como último recurso aunque por sí solas tiene un índice bajo de recuperación. Se deben realizar más estudios en cuanto a las terapias farmacológicas para la CT, ensayos clínicos controlados y cohortes de seguimiento, ya que son muy pocas las que existen. Los autores basados en la información encontrada tratan de dar una aproximación a la conducta frente a paciente con falla renal y CT, pero nunca tratan de ser una guía clínica ya que la evidencia no permite alguna aseveración de este tipo.

\section{Referencias:}

1. Giard A. Sur la calcificationhibernale. C R SocBiol 1898; 10: 1013-1015.

2. Duret MH. Tumoursmultiples et singulieres des boursessereuses (endotheliomes, peutetred'origineparasitaire). Bull Mem Soc Anat Paris 1899; 74: 725-733.

3. Teutschlaender O. Die lipoido-calcinosis oder lipoidkalkgicht. Beitr Pathol Ana 1949; 110: 402-432. 
4. Smack D, Norton SA, Fitzpatrick SE. Proposal for pathogenesis-based classification of tumoralcalcinosis. Int J Dermatol 1996; 35: 265-271.

5. Sprecher E. Familial TumoralCalcinosis: From Characterization of a Rare Phenotype to the Pathogenesis of Ectopic Calcification. J Inv Derm 2010; 130: 652-660.

6. Slavin RE, Wen J, Kumar D, Evans EB. Familial tumoralcalcinosis: a clinical, histopathologic, and ultrastructural study with an analysis of its calcifying process and pathogenesis. Am J Surg Pathol 1993; 17: 788-802.

7. Mockel G, Buttgereit F, Labs K, Perka C. Tumoralcalcinosis revisited: pathophysiology and treatment. Rheumatol Int 2005; 25: 55-9.

8. Horikoshi R, Akimoto T, Meguro D, Saito O, Ando Y, Muto S, Kusano E. Tumoralcalcinosis associated with hypercalcemia in a patient with chronic renal failure. Clin Exp Nephrol 2011; 15(1): 154-8.

9. Cancela AL, Oliveira RB, Graciolli FG, dos Reis LM. Fibroblast growth factor 23 in hemodialysis patients: effects of phosphate binder, calcitriol and calcium concentration in the dialysate. Nephron Clin Pract 2011; 117(1): c74-82.

10. Remy-Leroux V, Reguiaï Z, Labrousse AL, Zakine EM, Clavel P, Bernard P. Tumoralcalcinosis at an unusual site in a haemodialysis patient . Ann Dermatol Venereol 2009; 136(4): 350-4.

11. Tarrass F, Benjelloun M. Tumoralcalcinosis of the elbow in a long-term hemodialysis patient. Saudi J Kidney Dis Transpl 2008; 19(1): 105-6.

12. Topaz O, Shurman DL, Bergman R, et al. Mutations in GALNT3, encoding a protein involved in $\mathrm{O}$-linked glycosylation, cause familial tumoralcalcinosis. Nat Genet 2004; 36: 579-581.

13. Benet-Pages A, Orlik P, Strom TM, Lorenz- Depiereux B. An FGF23 missense mutation causes familial tumoralcalcinosis with hyperphosphatemia. Hum Mol Genet 2005; 14: 385-390.

14. Slavin RE, Wen J, Kumar D, Evans EB. Familial tumoralcalcinosis: a clinical, histopathologic, and ultrastructural study with an analysis of its calcifying process and pathogenesis. Am J Surg Pathol 1993; 17: 788-802.

15. Kim TH, Yang SY. Stomach calcification revealed by gastrofibroscopy in a haemodialysis patient. Nephrology 2010; 15(5): 592-3.

16. Clatworthy MR, Williams P, Watson CJ, Jamieson NV. The calcified abdominal cocoon. Lancet 2008; 371(9622): 1452

17. Okuda I, Ubara Y, Okuda C, Fujii T, Suwabe T, Kokubo T. A large calcified retroperitoneal mass in a patient with chronic renal failure: liposarcoma with ossification. Clin Exp Nephrol 2010; 14(2): 185-9.

18. Castillo-Lario MC, Carro-Alonso B, Jiménez-Ayllón E, Villavieja-Atance L. Diffuse metastatic calcifications caused by secondary hyperparathyroidism. Med Clin Barc 2007; 128(1): 40.

19. Kuo CW, Lee PT, Fang HC, Chou KJ, Chung HM, Chen CL. Extensive peritoneal calcification as a complication of long-term peritoneal dialysis and secondary hyperparathyroidism: a case report and review of the literature. Perit Dial Int 2006; 26(5): 609-12.

20. Nguyen VD, Nguyen KD, Kamath V. Unusual feature of soft-tissue calcification in chronic renal failure: tumoral calcification. Comput Med Imaging Graph 1991; 15(6): 397-402.

21. Francis DM, Busmanis I, Becker G. Peritoneal calcification in a peritoneal dialysis patient: a case report. Perit Dial Int 1990; 10(3): 237-40.

22. Yamada $\mathbf{H}$, Seto $\mathbf{K}$, Matsumoto $\mathbf{Y}$. A case of metastatic calcinosis of the oral cavity. J Oral Maxillofac Surg 2000; 58(3): 327-32.

23. Beerens AJ, Stel HV, Middelweerd MJ. Metastatic calciumphosphate deposition in the membranous nasal septum in end-stage renal disease. Rhinology 1999; 37(3): 136-8.

24. Gibson C, Robbins P, Guy S. Invasive uraemiccalcinosis of the hip. Clin Radiol 2009; 64(10): 1035-6.

25. Lin CJ, Wu CJ, Yeh JC, Chen YC, Chen HH. Unusual metastatic soft tissue calcification in a hemodialysis patient. South Med J 2008; 101(8): 851-3.

26. Ben Taarit C, el Younsi F, Turki S, Mestiri M,Zlitni M, Ben Maïz H. Tumorous calcinosis in hemodialysis: anatomo-clinical study apropos of 3 cases. Nephrologie 2001; 22(7): 349-52.

27. Minisola S, Romagnoli E, Rosso R. Massive tumoralcalcinosis in a patient on long-term hemodialysis. J Bone Miner Res 2000; 15(10): 2056-7.

28. García S, Cofán F, Combalia A, Casas A, Campistol JM, Oppenheimer F. Compression of the sciatic nerve in uremic tumor calcinosis. Neurologia 1999; 14(2): 86-9.

29. Apostolou T, Tziamalis M, Christodoulidou C, Fountas P, Billis A. Regression of massive tumoralcalcinosis of the ischium in a dialysis patient after treatment with reduced calcium dialysate and i.v. administration. Clin Nephrol 1998; 50(4): $247-51$

30. Gupta RK, Naran S, Lallu S, Fauck R, Johnston P. Fine needle aspiration cytology of soft tissue calcinosis presenting as an enlarging mass in the neck. Acta Cytol 2001; 45(3): 476-8.
31. Evans SE, Whitehouse GH. Extensive calcification in the breast in chronic renal failure. Br J Radiol 1991; 64(764): 757-9.

32. Hsu SC, Lee YY, Wang MC, Liu HL, Cheng MF, Huang JJ. Diffuse calcinosis and intradermal tophi in a uremic pateint: effect of low-calcium hemodialysis and mechanism of hypercalcemia. Blood Purif 2004; 22(2): 224-8.

33. Kinjo K, Yamashiro M, Akamine K, Kinjo M, Oshiro M. Diffuse hepatocellular calcification developing in a patient on chronic hemodialysis after ischemic hepatitis. Intern Med 2007; 46(20): 1729-33

34. Dubelaar IJ, Vissers E, Serafino G. Solid as a rock? Calcifications of the breast. Neth J Med 2010; 68(12): 425-430.

35. Castellanos MR, Paramanathan K, El-Sayegh S, Forte F, Buchbinder S, Kleiner M. Breast cancer screening in women with chronic kidney disease: the unrecognized effects of metastatic soft-tissue calcification. Nat Clin Pract Nephrol 2008; 4(6): 337-41.

36. Resnikoff LB, Mendelson EB, Tobin CE, Hendrix TM. Breast imaging case of the day.Metastatic calcification in the breast from secondary hyperparathyroidism induced by chronic renal failure. Radiographics 1996; 16(6): 1512-3.

37. Martín-Cuartero J, Yagüe-Romeo D, Abril-Avellanas E. Myocardial calcification in chronic renal failure. Rev Esp Cardiol 2007; 60(10): 1092

38. Marcu CB, Caracciolo E, Donohue T. Rapid progression of pericardial calcification in a patient with end-stage renal disease. Catheter Cardiovasc Interv 2005; $\mathbf{6 5}(\mathbf{1}): 43-6$

39. Basaria S, Kermani A. The heart of stone. Postgrad Med J 2001; 77(908): 405

40. Eguchi M, Tsuchihashi K, Takizawa H, Nakahara N, Hagiwara M, Ohnishi $\mathbf{H}$, et al. Detection of cardiac calcinosis in hemodialysis patients by whole-body scintigraphy with $99 \mathrm{~m}$-technetium methylene diphosphonate. Am J Nephrol 2000; 20(4): 278-82

41. Tsuchihashi K, Nozawa A, Marusaki S, Moniwa N, Oh-numa Y, Kuno A, et al. Mobile intracardiaccalcinosis: a new risk of thromboembolism in patients with haemodialysed end stage renal disease. Heart 1999; 82(5): 638-40.

42. Jing J, Kawashima A, Sickler K, Raval BK, Oldham SA. Metastatic cardiac calcification in a patient with chronic renal failure who was undergoing hemodialysis: radiographic and CT findings. AJR Am J Roentgenol 1998; 170(4): 903-5.

43. Fujimoto S, Hisanaga S, Yamatomo Y, Tanaka K, Hayashi T, Sumiyoshi A. Tertiary hyperparathyroidism associated with metastatic cardiac calcification in a haemodialyzed patient. Int Urol Nephrol 1991; 23(3): 285-92.

44. Restrepo J, Molina M. Tumoralcalcinosis of the knee. Acta Med Col 2010; 35: 185-186.

45. Coll B, Betriu A, Martínez-Alonso M, Amoedo ML, Arcidiacono MV, Borras M. Large artery calcification on dialysis patients is located in the intima and related to atherosclerosis. Clin J Am SocNephrol 2011; 6(2): 303-10.

46. Giardina F, Sudanese A, Bertoni F, Guerra E, Paderni S. Tumoral calcinosis of the popliteal space. Orthopedics 2004; 27(10): 1104-7.

47. Baums MH, Klinger HM, Otte S. MorbusTeutschländer - a massive soft-tissue calcification of the foot in a patient on long-term hemodialysis. Arch Orthop Trauma Surg 2003; 123(1): 51-3.

48. García S, Cofán F, Fernández de RP, Sala P, Oppenheimer F. Uremic tumoral calcinosis of the foot mimicking infection. Foot Ankle Int 2002; 23(3): 260-3.

49. De Alarcón RM, Palomares M, Marfil A, Asensio C. Posttransplant regression of uremic tumoralcalcinosis. Nefrología 2007; 27(3): 378-81.

50. Garg S, Sandur S, Buller GK. Dyspnea and left shoulder pain in an end-stage renal disease patient. Semin Dial 2007; 20(2): 172-3.

51. Tong MK, Siu YP. Tumoralcalcinosis in end stage renal disease. Postgrad Med J 2004; 80(948): 601.

52. Chow KM, Szeto CC, Wang AY, Li PK. Uraemictumouralcalcinosis. Nephrol Dial Transplant 2004; 19(2): 505-6.

53. Tsuchida K, Takemoto Y, Nakatani T, Sugimura K, Yamamoto K. Large soft tissue calcifications in a patient on short-term hemodialysis. Nephron 2002 90(2): 237-8.

54. García S, Cofán F, Combalia A, Campistol JM, Oppenheimer F, Ramón R. Compression of the ulnar nerve in Guyon's canal by uremic tumoralcalcinosis. Arch Orthop Trauma Surg 2000; 120(3-4): 228-30.

55. Borrás M, Marco MP, Belart M, Peña JM, Amoedo ML, Fernández E. Presentation of tumoralcalcinosis with systemic signs pointing to inflammatory disease. Nephrol Dial Transplant 1998;13(12): 3277-9

56. Lazowski P, Goldfarb DS. Images in clinical medicine. Calcified mass in a patient on long-term hemodialysis. N Engl J Med 1998; 338(20): 1427.

57. Franco M, Van Elslande L, Passeron C, Verdier JF, Barrillon D, CassutoViguier E. Tumoral calcinosis in hemodialysis patients. A review of three cases. Rev Rhum Engl Ed 1997; 64(1): 59-62.

58. Papadakis JT, Patrikarea A, Digenis GE, Stamatelou K, Ntaountaki I, Athanasopoulos V . Sodium thiosulfate in the treatment of tumoral calcifica- 
tions in a hemodialysis patient without hyperparathyroidism. Nephron 1996; 72(2): 308-12.

59. Canellos HM, Cooper J, Paek A, Chien J. Multiple calcified deposits along the eyelid margins secondary to chronic renal failure and hyperparathyroidism. Optometry 2005; 76(3): 181-4.

60. Cowlam TE, Bucknall TE. Cutaneous ectopic breast calcification in a haemodialysis patient. Breast 2003; 12(5): 342-4.

61. Reed MA, de Luna AM, Holaysan JS, Gerardo LT. Calcinosis cutis in chronic renal failure diagnosed by fine needle aspiration. A case report. Acta Cytol 2002; 46(4): 738-40.

62. Van Haren FM, Ruiter DJ, Hilbrands LB. Nadroparin-induced Calcinosis cutis in renal transplant recipients. Nephron 2001; 87(3): 279-82.

63. Enelow TJ, Huang W, Williams CM. Perforating papules in chronic renal failure. Metastatic calcinosis cutis with transepidermal elimination. Arch Dermatol 1998; 134(1): 98-9.

64. Solans EP, Bakhos R, Castelli MJ, Gattuso P. Fine needle aspiration biopsy of calcinosis cutis. A case report. Acta Cytol 1997; 41(2): 590-2.

65. Tan HH, Cheong WK. Cutaneous gangrene secondary to metastatic calcification in end stage renal failure--a case report. Singapore Med J 1996; 37(4): 438-40.

66. Omovie EE, Absi EG, Hill CM, Ezsias A, Potts AJ. Oral presentation of metastatic calcinosis cutis. Dentomaxillofac Radiol 1995; 24(3): 198-200.

67. Fox JG, Walli RK, Jaffray B, Simpson HK. Calcified subcutaneous nodules due to calcium heparin injections in a patient with chronic renal failure. Nephrol Dial Transplant 1994; 9(2): 187-8.

68. Tada J, Torigoe R, Shimoe K, Ohara S, Arata J, Ashizawa K. Calcium deposition in the skin of a hemodialysis patient with widespread skin necrosis. Am J Dermatopathol 1991; 13(6): 605-10.

69. Biswas A, Cooper J, Latifaj B. Metastatic calcinosiscutis presenting as bilateral vulval cysts. Br J Dermatol 2007; 157(3): 622-4

70. Thurley PD, Duerden R, Roe S, Pointon K. Case report: Rapidly progressive metastatic pulmonary calcification: evolution of changes on CT. Br J Radiol 2009; 82(980): e155-9

71. Golbin JM, Prakash UB, Blanco RE. A 57-year-old man with end-stage renal disease and chronic cough. Chest 2008; 133(4): 1021-4.

72. Low SY, Chau YP, Cheah FK. A 52-year-old man presenting with chronic cough and bilateral ground-glass opacities on CT of the thorax. Chest 2007; 132(4): 1401-5.

73. Block GA, Raggi P, Bellasi A, Kooienga L, Spiegel DM. Mortality effect of coronary calcification and phosphate binder choice in incident hemodialysis patients. Kidney Int 2007; 71(5): 438-41.

74. al-Homrany M, Grillo IA, al-Ghamdi B, el-Tahir M, Malatani TS, Khan MR. Diffuse pulmonary calcinosis and multiple soft tissue calcification in renal failure patient with pathological femoral fracture. Indian J Chest Dis Allied Sci 1998; 40(3): 205-11.

75. Carlson AP, Yonas HM, Turner PT. Disorders of tumoral calcification of the spine: illustrative case study and review of the literature. J Spinal Disord Tech 2007; 20(1): 97-103.

76. Dorenbeck U, Leingärtner T, Bretschneider T, Krämer BK, Feuerbach S. Tentorial and dural calcification with tertiary hyperparathyroidism: a rare entity in chronic renal failure. Eur Radiol 2002; 12 Suppl 3: S11-3.

77. Agarwal R, Burns RR, Vergne-Marini P. Paraparesis due to massive ectopic paravertebral calcification in a patient on maintenance hemodialysis. Am J Kidney Dis 1993; 22(5): 717-20.

78. Davies M, Griffiths P, White P, Donovan K. Tumour-like calcinosis causing reversible tetraparesis in a patient on continuous ambulatory peritoneal dialysis. Nephrol Dial Transplant 2001; 16(3): 622-4.

79. Martinez S, Vogler J, Harrelson J, Lyles K. Imaging of tumoral calcinosis: new observations. Radiology 1990, 174: 215-22.

80. Sahin A, Seven B, Yildirim M, Varoglu E. Heart and soft-tissue uptake of Tc-99m MDP in a hemodialysis patient. Clin Nucl Med 2004; 29(12): 854-5.

81. Cesani F, Villanueva-Meyer J. Intense myocardial and lung uptake of $99 \mathrm{~m}-\mathrm{Tc}-$ pyrophosphate using single photon emission computed tomography in a patient with end-stage renal disease and secondary hyperparathyroidism. Int Urol Nephrol 1996; 28(4): 569-74.

82. Brodeur FJ Jr, Kazerooni EA. Metastatic pulmonary calcification mimicking airspace disease. Technetium-99m-MDP SPECT imaging. Chest 1994; 106(2): 620-2.

83. Ghacha R, Sinha AK, Karkar AM. Spontaneous resolution of extensive periarticular metastatic calcification after renal transplant in a case of end stage renal disease. Ren Fail 2002; 24(2): 239-44.

84. Rovno HD, Feig SA, Hughes JS, Hurford MT, Karasick D, Filippone E. Breast imaging case of the day. Spontaneously disappearing benign calcifications of the breast. Radiographics 1998; 18(6): 1599-604.

85. Schwarz A, Merkel S, Leitolf H, Haller H. The effect of cinacalcet on bone remodeling and renal function in transplant patients with persistent hyperparathyroidism. Transplantation 2011; 91(5): 560-5.

86. Walsh M, Manns BJ, Klarenbach S, Tonelli M, Hemmelgarn B, Culleton B. The effects of nocturnal compared with conventional hemodialysis on mineral metabolism: A randomized-controlled trial. Hemodial Int 2010; 14(2): 174-81.

87. PhanischM,Ravanan R, Lawsom T, Baboolal K. Tumoralcalcinosis associated with pyrexia and systemic inflammatory response in a haemodialysis patient: successful treatment using intravenous pamidronate. Nephrol Dial Transplant 2000; 15(10): 1691-1693.

88. Mori H, Okada Y, Tanaka Y. Etidronate for the treatment of progressive tumoralcalcinosis in hemodialysis patients. Intern Med 2007; 46(17): 1485-6.

89. Jacob JJ, Mathew K, Thomas N. Idiopathic sporadic tumoral calcinosis of the hip: successful oral bisphosphonate therapy. Endocr Pract 2007; 13(2): 182-6.

90. Ebenbichler GR, Erdogmus CB, Resch KL, et al. Ultrasound therapy for calcific tendinitis of the shoulder. N Engl J Med 1999; 340: 1533-1538.

91. Hsu CH. Historical perspective on management of calcium and phosphorus metabolism in chronic renal failure. Am J Kidney Dis 2001; 37(1): 195-201. 